\title{
Glycemic Control in the Medical Intensive Care Unit
}

\author{
Michelle A. Kovalaske, M.D., and Gunjan Y. Gandhi, M.D., M.Sc.
}

\begin{abstract}
Hyperglycemia in the critically ill is a well-known phenomenon, even in those without known diabetes. The stress response is due to a complex interplay between counter-regulatory hormones, cytokines, and changes in insulin sensitivity. Illness/infection, overfeeding, medications (e.g., corticosteroids), insufficient insulin, and/or volume depletion can be additional contributors. Acute hyperglycemia can adversely affect fluid balance (through glycosuria and dehydration), immune and endothelial function, inflammation, and outcome. While there are several insulin infusion protocols that are able to safely and effectively treat hyperglycemia, the bulk of accumulated evidence does not support a causal relationship between acute hyperglycemia and adverse outcomes in the medical intensive care unit. Meta-analysis of randomized controlled trials suggests there is no benefit to tightening glucose control to normal levels compared to a reasonable and achievable goal of 140 to $180 \mathrm{mg} / \mathrm{dl}$. There is a significantly increased risk of hypoglycemia. Although there is some evidence that patients without known diabetes have worse outcomes than those with known diabetes, more recent evidence is conflicting. Glycemic control in critically ill patients should not be neglected, as studies have not tested tight versus no/poor control, but tight versus good control. A moderate approach to managing critical illness hyperglycemia seems most prudent at this juncture. Future research should ascertain whether there are certain subgroups of patients that would benefit from tighter glycemic goals. It also remains to be seen if tight glucose control is beneficial once hypoglycemia is minimized with technological advances such as continuous glucose monitoring systems.
\end{abstract}

J Diabetes Sci Technol 2009;3(6):1330-1341

\section{Introduction}

$\mathrm{B}_{\mathrm{e}}$ This changed dramatically when tight glycemic control came to the forefront of hospital medicine after a landmark randomized controlled trial (RCT) (which has been cited over 2200 times) reported a $42 \%$ reduction in mortality in surgical intensive care unit (ICU) patients when euglycemia was achieved $(80-110 \mathrm{mg} / \mathrm{dl}) .{ }^{1}$ Since then, massive efforts have been made worldwide to achieve these goals, with intensive insulin therapy (IIT) becoming a benchmark in critically ill patients. Clinicians realized there are significant challenges to achieving such ambitious goals in practice, namely, multifold increased risk of hypoglycemia, increased utilization of resources, significant revamping of infrastructure to implement glycemic management protocols, additional personnel training, and need for intense coordination among

Author Affiliation: Mayo Clinic Division of Endocrinology and Metabolism, Jacksonville, Florida

Abbreviations: (ADA) American Diabetes Association, (CI) confidence interval, (GLUT) glucose transporter (ICU) intensive care unit, (IIT) intensive insulin therapy, (LOS) length of stay, (NICE-SUGAR) Normoglycaemia in Intensive Care Evaluation Survival Using Glucose Algorithm Regulation, (NNH) number needed to harm, (OR) odds ratio, (RCT) randomized controlled trial

Keywords: critically ill, glycemic control 
varied involved specialties. While laudable efforts have been undertaken, from tertiary care academic centers to small community hospitals, for seamless implementation of insulin protocols, subsequent clinical studies in varied ICU settings could not replicate the amazing benefits. The much-awaited Normoglycaemia in Intensive Care Evaluation Survival Using Glucose Algorithm Regulation (NICE-SUGAR) trial results provide definitive and not entirely surprising evidence of what has thus been suspected: there is no benefit of tightening glucose control to normal levels compared to a reasonable and achievable goal of 140 to $180 \mathrm{mg} / \mathrm{dl}$. Professional organizations such as the American Association of Clinical Endocrinologists and the American Diabetes Association (ADA) have come full circle on this issue, now recommending a more moderate approach to treating hyperglycemia in the critically ill. In this article focused on hyperglycemia in the medical ICU, we discuss the pathophysiology and etiology of stress hyperglycemia, review the current evidence linking hyperglycemia and outcomes, and attempt to delineate the contribution of diabetes to outcomes.

\section{Epidemiology}

Hyperglycemia in the critically ill is a common phenomenon. Its prevalence is difficult to estimate, because the diagnosis is variably defined. For instance, in one study that attempted to measure the prevalence of hyperglycemia in the medical ICU of a tertiary care medical center, more than $60 \%$ of patients had a blood glucose greater than $110 \mathrm{mg} / \mathrm{dl}$ after ICU admission, 38\% greater than $150 \mathrm{mg} / \mathrm{dl}$, and $23 \%$ greater than $200 \mathrm{mg} / \mathrm{dl}^{2}$ Only one in five of these patients had a history of known diabetes. This estimate of prevalence of diabetes is consistent with patients who undergo cardiac surgery. ${ }^{3}$ Thus the vast majority of patients in the ICU have stress hyperglycemia, which refers to transient hyperglycemia during illness and is usually restricted to patients without previous evidence of diabetes. ${ }^{4}$ One proposed definition in this group of patients is the ADA criteria of fasting plasma glucose $>125 \mathrm{mg} / \mathrm{dl}$ or random plasma glucose $>200 \mathrm{mg} / \mathrm{dl}$. In patients with preexisting diabetes, stress hyperglycemia manifests as a deterioration of pre-illness glycemic control.

\section{Pathophysiology of Hyperglycemia}

The homeostatic mechanisms that maintain euglycemia in the postabsorptive state and limit the postprandial glucose increase in subjects without diabetes are impaired in patients with diabetes. Patients with diabetes have decreased insulin secretion and/or action resulting in preprandial and postprandial hyperglycemia due to increased hepatic glucose production and decreased glucose uptake. Severe stress can cause hyperglycemia in patients without a prior diagnosis of diabetes. This degree of stress (as during a serious illness) likely has a different pathophysiologic mechanism than type 1 or type 2 diabetes. The stress response is a complex interplay of counter-regulatory hormones, cytokines, and changes in insulin sensitivity (Figure 1). Glucagon, epinephrine, cortisol, growth hormone, and norepinephrine increase gluconeogenesis and glycogenolysis, thereby increasing glucose production. ${ }^{4}$ Tumor necrosis factor-alpha may also contribute by increasing gluconeogenesis and plasma glucagon. ${ }^{5}$ Insulin production is also increased, but sepsis and critical illness impair the insulin signaling pathway, causing decreased glucose transporter (Glut)-4 mediated glucose uptake, which in turn leads to insulin resistance. ${ }^{6}$ Noninsulin-mediated uptake occurs by cytokine upregulation of Glut-1, 1,8 which causes increased oxidative metabolism and decreased nonoxidative metabolism. Stress causes an even greater derangement in glucose metabolism in patients with diabetes, because they cannot increase insulin secretion as a compensatory response. The exaggerated glucose response observed following stress dose counter-regulatory hormone infusion in otherwise healthy subjects with diabetes compared with subjects without diabetes helps explain why glucose control frequently deteriorates in ill diabetes patients. ${ }^{9}$

\section{Hyperglycemia and Hypoglycemia: Common Causes}

Clinicians should be familiar with the causes of exogenous hyperglycemia and hypoglycemia to minimize events or recurrence. ${ }^{10,11}$ Illness/infection, overfeeding (nutrition support, dextrose-containing crystalloid, dextrose absorption during peritoneal dialysis, and medications formulated in fat emulsion, such as propofol), medications (e.g., corticosteroids, sympathomimetic infusions, or immunosuppressants), insufficient insulin, and/or volume depletion can cause hyperglycemia. Since unexplained hyperglycemia can be a harbinger of infection, central catheters should be considered as a source of infection. Even bed rest alone may contribute by causing reduced insulin sensitivity of skeletal muscle. ${ }^{12}$ Causes of hypoglycemia include excess insulin dose, unanticipated discontinuation of nutrition support in insulin-treated patients, resolution of severe stress, discontinuation or decreased doses of corticosteroids or sympathomimetic agents, renal dysfunction, severe hepatitis, sepsis, and diabetic gastroparesis. 


\section{Adverse Effects of Acute Hyperglycemia}

During short-term hospitalization, hyperglycemia can adversely affect fluid balance (through glycosuria and dehydration), immune function, ${ }^{13,14}$ inflammation, and outcome. In vitro studies report that hyperglycemia is associated with abnormalities in white cell function, including granulocyte adhesion, chemotaxis, phagocytosis, respiratory burst, superoxide formation, and intracellular killing. Hyperglycemia can also impair complement

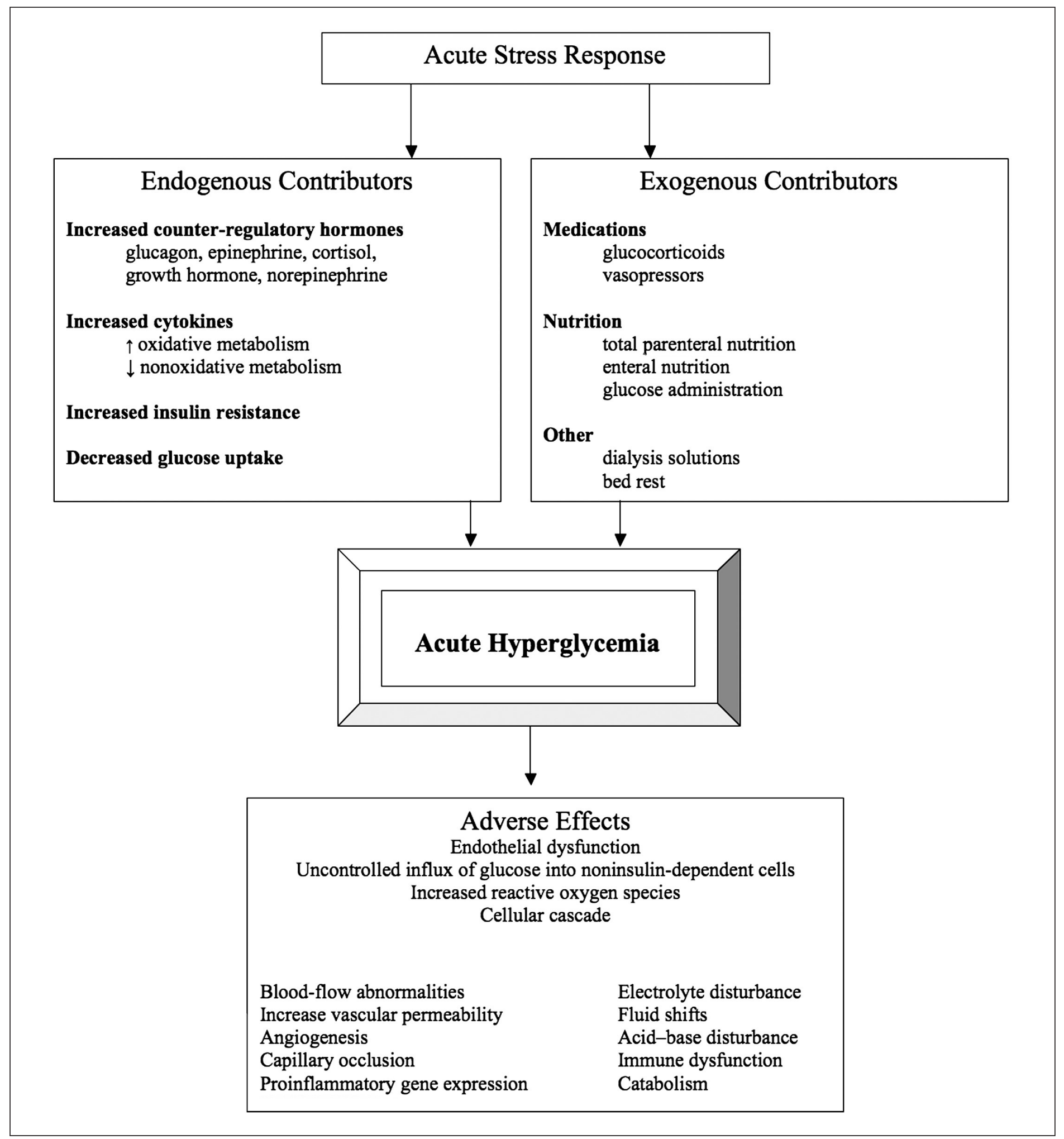

Figure 1. Etiology of acute hyperglycemia and adverse tissue effects. Information modified from the study of Dungan et al. ${ }^{4}$ 
activity. Glucose, through complement glycation, has the potential to compete with microorganisms for the attachment of complement, thereby inhibiting opsonization. ${ }^{15-17}$ These abnormalities improve with glucose control. Acute hyperglycemia is also known to cause endothelial cell dysfunction. It is thought that the stressful environment decreases the ability of the noninsulin-mediated Glut-1 transporter to down regulate, which is the normal physiologic protective mechanism to hyperglycemia. This causes an uncontrolled influx of high levels of glucose into the cell, leading to a vicious cycle of up-regulation of more Glut-1 transporters. ${ }^{18}$ Hyperglycemia within the cell increases production of reactive oxygen species, which then creates a cascade of cellular effects, increasing polyol pathway influx, advanced glycation end products, NFKB, and hexosamine pathway. ${ }^{19}$ These downstream effects lead to blood-flow abnormalities, increased vascular permeability, angiogenesis, capillary occlusion, and pro-inflammatory gene expression. ${ }^{20}$ Most of this work has been done to describe the mechanisms behind chronic complications of diabetes, but in critical illness, there is evidence that wholebody glucose uptake is increased, specifically by tissues that are not insulin dependent. ${ }^{21}$ Increased catabolism, lipotoxicity, sympathetic nervous system activation, and extracellular matrix deposition also contribute to tissue effects of hyperglycemia. ${ }^{4}$

\section{Outcomes with Intensive Insulin Therapy in the Medical Intensive Care Unit}

There have been six RCTs for patients in a dedicated medical ICU attempting to evaluate IIT (Table 1). Two evaluated patients with acute cerebrovascular accident. $^{22,23}$ Oksanen and colleagues studied patients who had ventricular fibrillation outside the hospital with subsequent cardiopulmonary resuscitation and hypothermia treatment. ${ }^{24}$ The other studies encompassed many nonsurgical critical medical illnesses. ${ }^{25-27}$ Of the total 1460 patients, 1200 were contributed by the largest trial done in Leuven, Belgium. ${ }^{25,26}$ The percentage of diabetes patients involved in each study varied greatly and ranged from $11 \%$ to $89 \%$. Most studies occurred at a single site and were unblinded RCTs with the exception of one study, ${ }^{22}$ in which acute intervention was blinded to patients and families. The majority of these studies were not blinded, because the treating providers needed to know the goal blood glucose to make appropriate insulin adjustments. Also, the most common primary endpoint was mortality, a solid endpoint less subject to bias. The goal for intensively treated patients ranged from 72 to $144 \mathrm{mg} / \mathrm{dl}$, a fairly narrow range, but the goals for the control group varied greatly. All patients (both treatment and control groups) received insulin therapy via intravenous infusion according to a nursedriven protocol, with the exception of one study in which the control group used subcutaneous insulin. ${ }^{22}$

The results of each study showed a statistically significant difference in blood glucose measurements between the intensive and control groups. However, none of the studies-including the largest study_-showed a significant reduction in mortality with IIT, except in a subgroup of patients who stayed in the ICU for 3 or more days. In this subgroup, the intensively treated group had a significant improvement in survival in the ICU and in the hospital compared to the conventionally treated group (31\% versus $38 \%, p=.05$, and $43 \%$ versus $52 \%$, $p=.02$, respectively). ${ }^{26}$ Morbidity was significantly reduced by the prevention of newly acquired kidney injury, accelerated weaning from mechanical ventilation, and accelerated discharge from the ICU and hospital. In a published meta-analysis, ${ }^{28}$ pooled data from these six RCTs revealed a risk ratio of 1.00 (95\% confidence interval [CI], 0.78 to 1.28 ), which suggests that IIT in the medical ICU does not reduce mortality. Hypoglycemia is obviously the major adverse event and was increased in all studies. There is one other important aspect of patient care that should be mentioned: nutrition. How these patients were fed certainly could contribute to hyperglycemia, and whether total parenteral nutrition, tube feedings, or oral intake was allowed is yet another variable to consider when comparing results of these trials. The study by Van den Berghe and associates is really the only one of the six that clearly delineates nutrition. ${ }^{26}$ Each of these patients received enteral feeding of $22-30 \mathrm{kcal} / \mathrm{kg} / 24 \mathrm{~h}$ as soon as they were stable. Several limitations of the trial performed at a single center in Belgium-including the unusual practice of early initiation of nutrition resulting in significant hyperglycemia in the control group, liberal definition of hypoglycemia (less than $40 \mathrm{mg} / \mathrm{dl}$ rather than less than $60 \mathrm{mg} / \mathrm{dl}$ ), and lack of external validity among others-have been reported. ${ }^{2}$

\section{Outcomes with Intensive Insulin Therapy in Mixed Medical-Surgical Intensive Care Units}

A summary of seven of the major RCTs in mixed medicalsurgical ICUs is listed in Table 2. ${ }^{29-35}$ NICE-SUGAR was the largest international study to date on the topic, accounting for more than $67 \%$ of the 8965 patients in these seven trials. ${ }^{34}$ The NICE-SUGAR trial tries to 
answer the question of whether intensive glucose control (goal 81 to $108 \mathrm{mg} / \mathrm{dl}$ ) reduces 90-day mortality in critically ill adults compared to conventional treatment (144 to $180 \mathrm{mg} / \mathrm{dl}$ ). It was conducted in 42 ICUs in North America, Australia, and New Zealand and included 6104 patients; $62 \%$ (3791/6029 with available data) were nonsurgical, making it by far the largest RCT to provide data on medical ICU patients. The two treatment groups showed good glycemic separation with a difference of
$29 \mathrm{mg} / \mathrm{dl}$ in glucose levels (as compared to $42 \mathrm{mg} / \mathrm{dl}$ in the Leuven medical ICU study). The results differ from other trials in that there was an increase in the primary endpoint (death at 90 days) with intensive glucose control (28\% versus $25 \%$ with conventional control: odds ratio [OR] 1.14; 95\% CI, 1.02 to 1.28; number needed to harm [NNH] 39). Treatment effect did not differ significantly between operative and medical patients (OR of death in the intensive control group 1.31 and 1.07, respectively).

Table 1 .

Summary of Randomized Controlled Trials in Dedicated Medical Intensive Care Units

\begin{tabular}{|c|c|c|c|c|c|c|}
\hline & Bland et al. ${ }^{27}$ & $\begin{array}{c}\text { Van den Berghe } \\
\text { et al. } .50\end{array}$ & Walters et al. ${ }^{23}$ & Farah et al. ${ }^{25}$ & $\begin{array}{l}\text { Oksanen } \\
\text { et al. }{ }^{24}\end{array}$ & Bruno et al. ${ }^{22}$ \\
\hline Study design & $\begin{array}{l}\text { Unblinded, } \\
\text { single center }\end{array}$ & $\begin{array}{l}\text { Unblinded, } \\
\text { single center }\end{array}$ & $\begin{array}{l}\text { Unblinded, } \\
\text { single center }\end{array}$ & $\begin{array}{l}\text { Unblinded, } \\
\text { single center }\end{array}$ & $\begin{array}{l}\text { Unblinded } \\
2 \text { ICUs }\end{array}$ & $\begin{array}{c}\text { Single/double } \\
\text { blinded, multicenter }\end{array}$ \\
\hline $\begin{array}{l}\text { Number of patients } \\
(\mathrm{M} / \mathrm{F})\end{array}$ & $\begin{array}{c}10 \\
(7 / 3)\end{array}$ & $\begin{array}{c}1200 \\
(738 / 462) \\
\end{array}$ & $\begin{array}{c}25 \\
(10 / 15)\end{array}$ & $\begin{array}{c}89 \\
(46 / 43) \\
\end{array}$ & $\begin{array}{c}90 \\
(71 / 19) \\
\end{array}$ & $\begin{array}{c}46 \\
(26 / 20)\end{array}$ \\
\hline Mean age, years & 57 & 63 & 75 & 73 & 64 & 58 \\
\hline City, country & $\begin{array}{l}\text { California, } \\
\text { U.S.A. }\end{array}$ & $\begin{array}{l}\text { Leuven, } \\
\text { Belgium }\end{array}$ & $\begin{array}{l}\text { Glasgow, } \\
\text { UK }\end{array}$ & $\begin{array}{l}\text { Nahariya, } \\
\text { Israel }\end{array}$ & $\begin{array}{l}\text { Helsinki, } \\
\text { Finland }\end{array}$ & $\begin{array}{l}\text { Multicenter, } \\
\text { U.S.A. }\end{array}$ \\
\hline $\begin{array}{l}\text { Number with } \\
\text { diabetes (\%) }\end{array}$ & $4(40 \%)$ & 203 (16.9\%) & $13(52 \%)$ & $53(59 \%)^{a}$ & $10(11 \%)$ & 42 (91\%) \\
\hline Illness & $\begin{array}{l}\text { Had to require } \\
\text { intubation }\end{array}$ & Medical ICU & $\begin{array}{c}\text { Acute } \\
\text { cerebrovascular } \\
\text { accident }\end{array}$ & Medical ICU & $\begin{array}{l}\text { Ventricular } \\
\text { fibrillation } \\
\text { event out of } \\
\text { hospital/revived/ } \\
\text { hypothermic } \\
\text { protocol }\end{array}$ & $\begin{array}{c}\text { Acute } \\
\text { cerebrovascular } \\
\text { accident }\end{array}$ \\
\hline Patient-to-nurse ratio & $2: 1$ or $1: 1$ & $1: 2.5$ & Not reported & Not reported & $1: 1,2: 1$ & Not reported \\
\hline $\begin{array}{l}\text { Blood glucose } \\
\text { sampling }\end{array}$ & $\begin{array}{c}\text { Capillary or } \\
\text { arterial }\end{array}$ & Capillary or arterial & Capillary & Not reported & Not reported & Capillary \\
\hline Frequency of testing & $\begin{array}{l}\text { Every hour until } \\
4 \text { in target then } \\
\text { every } 2 \mathrm{~h}\end{array}$ & $\begin{array}{l}\text { Every } 4 \mathrm{~h} \text { unless } \\
\text { nurse felt need for } \\
\text { more frequent }\end{array}$ & $\begin{array}{c}\text { Every } 2 \mathrm{~h} \text { for } \\
48 \mathrm{~h}\end{array}$ & Every $4 \mathrm{~h}$ & $\begin{array}{c}\text { Every } 30 \text { min until } \\
\text { target, then every } \\
4 \mathrm{~h} \text { at least for } 48 \\
\text { hours }\end{array}$ & $\begin{array}{l}\text { Every hour, intensive; } \\
\text { every } 2 \text { h, control }\end{array}$ \\
\hline Type of insulin & $\begin{array}{l}\text { Intravenous } \\
\text { insulin }\end{array}$ & $\begin{array}{l}\text { Intravenous } \\
\text { insulin }\end{array}$ & $\begin{array}{l}\text { Intravenous } \\
\text { insulin }\end{array}$ & $\begin{array}{l}\text { Intravenous } \\
\text { insulin }\end{array}$ & $\begin{array}{l}\text { Intravenous } \\
\text { insulin }\end{array}$ & $\begin{array}{c}\text { Intensive, } \\
\text { intravenous insulin + } \\
\text { subcutaneous saline } \\
\text { four times per day. } \\
\text { Control, } \\
\text { intravenous saline + } \\
\text { subcutaneous insulin } \\
\text { four times per day. } \\
\text { Both, subcutaneous } \\
\text { insulin once eating }\end{array}$ \\
\hline Feeding & $\begin{array}{l}\text { If no total } \\
\text { parenteral } \\
\text { nutrition or } \\
\text { tube feeding, } \\
\text { received D5 at } \\
50 \mathrm{ml} / \mathrm{h}\end{array}$ & $\begin{array}{c}\text { Enteral feeding } \\
22-30 \mathrm{kcal} / \mathrm{kg} / 24 \mathrm{~h} \\
\text { as soon as stable }\end{array}$ & $\begin{array}{c}\text { Standard } \\
\text { hospital diet, } \\
\text { no tube feeding }\end{array}$ & Not described & Not described & Eating after $24 \mathrm{~h}$ \\
\hline $\begin{array}{l}\text { Target blood glucose, } \\
\text { intensive (mg/dl) }\end{array}$ & $80-110$ & $80-110$ & $90-144$ & $110-140$ & $72-108$ & $90-130$ \\
\hline
\end{tabular}


Table 1. Continued

\begin{tabular}{|c|c|c|c|c|c|c|}
\hline & Bland et al. ${ }^{27}$ & $\begin{array}{c}\text { Van den Berghe } \\
\text { et al. } .^{50}\end{array}$ & Walters et al. ${ }^{23}$ & Farah et al. ${ }^{25}$ & Oksanen et al. ${ }^{24}$ & Bruno et al. ${ }^{22}$ \\
\hline $\begin{array}{l}\text { Target blood glucose, } \\
\text { control (mg/dl) }\end{array}$ & $180-200$ & $180-200$ & $<270$ & $140-200$ & $108-144$ & $<200$ \\
\hline $\begin{array}{l}\text { Mean blood glucose, } \\
\text { intense (mg/dl) }\end{array}$ & 105 & $\begin{array}{l}111 / 108 \\
\text { (>3 days) }\end{array}$ & $\begin{array}{l}\text { Significant decrease } \\
\text { in mean area under } \\
\text { glucose time curve: } \\
324 \mathrm{~h} \cdot \mathrm{mmol} / \mathrm{liter}\end{array}$ & 142 & 90 & 133 \\
\hline $\begin{array}{l}\text { Mean blood glucose, } \\
\text { control (mg/dl) }\end{array}$ & 177 & $\begin{array}{l}\text { 153/156 } \\
\text { (>3 days) }\end{array}$ & $385 \mathrm{~h} \cdot \mathrm{mmol} / \mathrm{liter}$ & 174 & 115 & 190 \\
\hline APACHE II & Not listed & $\sim 23$ & Not listed & $\sim 22$ & $\sim 25$ & Not listed \\
\hline Primary endpoint & $\begin{array}{l}\text { 28-day } \\
\text { mortality }\end{array}$ & $\begin{array}{l}\text { In hospital } \\
\text { mortality }\end{array}$ & $\begin{array}{l}\text { 28-day } \\
\text { mortality }\end{array}$ & $\begin{array}{l}\text { 28-day } \\
\text { mortality }\end{array}$ & $\begin{array}{l}\text { 30-day } \\
\text { mortality }\end{array}$ & $\begin{array}{l}\text { 90-day } \\
\text { mortality }\end{array}$ \\
\hline Mortality, intensive & $\begin{array}{l}1 / 5 \text { (not } \\
\text { powered) }\end{array}$ & $182(30 \%)$ & $1 / 13(7.6 \%)$ & $22(53.6 \%)$ & $33 \%$ & $2(7 \%)$ \\
\hline Mortality, control & $\begin{array}{c}2 / 5 \\
\text { (not powered) }\end{array}$ & $178(29.9 \%)$ & 0/12 (0\%) & $22(45 \%)$ & $\begin{array}{c}35 \% \\
(p=.846)\end{array}$ & 0 \\
\hline $\begin{array}{l}\text { Mean LOS, intensive } \\
\text { (days) }\end{array}$ & 32 & 13 & Not reported & 7 & Not reported & Not reported \\
\hline $\begin{array}{l}\text { Mean LOS, control } \\
\text { (days) }\end{array}$ & 32 & 13 & Not reported & 7 & Not reported & Not reported \\
\hline $\begin{array}{l}\text { Hypoglycemic events } \\
<40 \mathrm{mg} / \mathrm{dl} \text {, intensive }\end{array}$ & $\begin{array}{c}2 \text { events } \\
(0.1 \%)\end{array}$ & $\begin{array}{c}111(18.7 \%) / \\
97(25.1 \%)\end{array}$ & 0 & $\begin{array}{c}0.56 \text { events/ } \\
\text { patient }\end{array}$ & 0 & $\begin{array}{l}\text { Defined as } \\
<60 \mathrm{mg} / \mathrm{dl}\end{array}$ \\
\hline $\begin{array}{l}\text { Hypoglycemic events } \\
<40 \mathrm{mg} / \mathrm{dl} \text {, control }\end{array}$ & $\begin{array}{l}2 \text { events } \\
(0.1 \%)\end{array}$ & $\begin{array}{l}19(3.1 \%) / \\
15(3.9 \%)\end{array}$ & 0 & $\begin{array}{l}0.48 \text { events/ } \\
\text { patient }\end{array}$ & 0 & $\begin{array}{l}\text { Defined as } \\
<60 \mathrm{mg} / \mathrm{dl}\end{array}$ \\
\hline $\begin{array}{l}\text { Hypoglycemic events } \\
40-60 \mathrm{mg} / \mathrm{dl} \text {, intensive }\end{array}$ & $(2.1 \%)$ & Not assessed & 0 & $\begin{array}{l}\text { Hypoglycemia } \\
\text { not defined }\end{array}$ & $(18 \%)$ & $\begin{array}{l}11(35 \%), \\
\text { all events } \\
<60 \mathrm{mg} / \mathrm{dl}\end{array}$ \\
\hline $\begin{array}{l}\text { Hypoglycemic events } \\
40-60 \mathrm{mg} / \mathrm{dl} \text {, control }\end{array}$ & $(0.2 \%)$ & Not assessed & 0 & $\begin{array}{l}\text { Hypoglycemia } \\
\text { not defined }\end{array}$ & $2 \%$ & $\begin{array}{l}0, \text { all events } \\
<60 \mathrm{mg} / \mathrm{dl}\end{array}$ \\
\hline
\end{tabular}

Severe hypoglycemia occurred in more patients in the intensive control group compared to the conventional control group (7\% versus $1 \%$; relative risk increase $1272 \%$; $95 \%$ CI, $720 \%$ to $2200 \%$; NNH 16). Although not shown by available data, unrecognized hypoglycemia may very well be the major culprit for the increased mortality, especially in critically ill patients sedated and on a mechanical ventilator. The relationship between hyperglycemia in the critically ill (the majority not having underlying diabetes) and worse outcomes in most previous observational studies is not causal, but a reflection of the severity of illness. Thus attempting to reverse a normal stress response of shunting energy to critical organs may very well be deleterious. Among the other trials, two focused on severe sepsis. ${ }^{31,35}$ One of these studies was stopped early, secondary to its high incidence of hypoglycemia. ${ }^{31}$ There was no significant reduction in mortality in the trials that did assess it as the primary outcome.

\section{Outcomes from Meta-Analyses}

A meta-analysis by Griesdale and coworkers ${ }^{28}$ incorporates NICE-SUGAR and the two large Leuven studies among many other smaller studies to attempt to resolve the disparate results and answer the question of whether we are helping or hurting patients with tight glycemic control. The NICE-SUGAR study contributed almost half of the patients in the analysis; they were quite ill, with mortality rates approaching 30\%. Over 2200 of the patients included in NICE-SUGAR had undergone surgery, but mostly emergency surgery, unlike the Belgian surgical ICU study. Also, the separation between morning glucose levels was more modest with NICE-SUGAR (27 versus $47 \mathrm{mg} / \mathrm{dl}$ ). While the surgical ICU RCT by Van den Berghe and colleagues found mortality benefit only in patients treated with insulin infusion for more than 5 days, the median duration of treatment in NICE-SUGAR was 4 days. A RCT conducted by Van den Berghe and 
associates was large enough to swing the results in the subgroup of surgical ICUs toward a mortality benefit. Whether patients post-elective surgery, especially cardiac surgery, are significantly different (i.e., follow the acutereperfusion model) and benefit from IIT remains to be seen. However, the outcome of the pooled data for these 13,000 critically ill patients tells us that the truth may lie somewhere in between, and tight glycemic control may not actually have any effect on mortality at all.

Table 2.

Summary of Major Randomized Controlled Trials in Mixed Intensive Care Units

\begin{tabular}{|c|c|c|c|c|c|c|c|}
\hline & Arabi et al. ${ }^{29}$ & $\begin{array}{l}\text { Brunkhorst } \\
\text { et al. }{ }^{31 a}\end{array}$ & $\begin{array}{l}\text { NICE- } \\
\text { SUGAR }^{34}\end{array}$ & $\begin{array}{l}\text { Azevedo } \\
\text { et al. }{ }^{30}\end{array}$ & $\begin{array}{l}\text { Devos } \\
\text { et al. }{ }^{33}\end{array}$ & lapichino et al. ${ }^{35}$ & $\begin{array}{c}\text { De La Rosa Gdel } \\
\text { et } a l .^{32}\end{array}$ \\
\hline Study design & $\begin{array}{l}\text { Unblinded } \\
\text { single center }\end{array}$ & $\begin{array}{l}\text { Unblinded } \\
\text { multicenter }\end{array}$ & $\begin{array}{l}\text { Unblinded } \\
\text { international } \\
\text { multicenter }\end{array}$ & $\begin{array}{l}\text { Unblinded } \\
\text { multicenter }\end{array}$ & $\begin{array}{l}\text { Unblinded } \\
\text { multicenter }\end{array}$ & $\begin{array}{l}\text { Unblinded } \\
\text { multicenter }\end{array}$ & $\begin{array}{l}\text { Unblinded } \\
\text { single center }\end{array}$ \\
\hline $\begin{array}{l}\text { Number of patients } \\
(\mathrm{M} / \mathrm{F})\end{array}$ & $\begin{array}{c}523 \\
(357 / 132)\end{array}$ & $\begin{array}{c}488 \\
(322 / 215)\end{array}$ & $\begin{array}{c}6030 \\
(4023 / 2207)\end{array}$ & 337 & $\begin{array}{c}1011 \\
(634 / 377)\end{array}$ & $\begin{array}{c}72 \\
(47 / 25)\end{array}$ & $\begin{array}{c}504 \\
(301 / 203)\end{array}$ \\
\hline Mean age, years & 52.5 & 64.6 & 60 & Adults & 65 & 62.3 & 46.6 \\
\hline City, country & $\begin{array}{c}\text { Riyadh, } \\
\text { Saudi Arabia }\end{array}$ & $\begin{array}{l}\text { Multicity, } \\
\text { Germany }\end{array}$ & International & $\begin{array}{l}\text { Sao Luiz - } \\
\text { MA, Brazil }\end{array}$ & 7 countries & $\begin{array}{l}\text { Milan, } \\
\text { Italy }\end{array}$ & $\begin{array}{l}\text { Medellin, } \\
\text { Colombia }\end{array}$ \\
\hline Medical ICU patients & $\begin{array}{c}83 \% \\
(435 / 523)\end{array}$ & $47 \%$ & $\begin{array}{c}63 \% \\
(3796 / 6029)\end{array}$ & Not defined & $41 \%$ & $63 \%$ & $\begin{array}{c}49 \% \\
(246 / 504)\end{array}$ \\
\hline $\begin{array}{l}\text { Number with } \\
\text { diabetes (\%) }\end{array}$ & $\begin{array}{c}208 \\
(39 \%)\end{array}$ & $\begin{array}{c}163 \\
(30 \%)\end{array}$ & $\begin{array}{c}1211 / 6029 \\
(20 \%)\end{array}$ & Not reported & $\begin{array}{l}\text { Not } \\
\text { reported }\end{array}$ & $\begin{array}{l}12 / 72 \\
(17 \%)\end{array}$ & $\begin{array}{c}61 / 504 \\
(12 \%)\end{array}$ \\
\hline Illness & Mixed ICU & Severe sepsis & Mixed ICU & Mixed ICU & Mixed ICU & Severe sepsis & Mixed ICU \\
\hline $\begin{array}{l}\text { Patient- to-nurse } \\
\text { ratio }\end{array}$ & $1.2: 1$ & Not reported & $\begin{array}{l}\text { Not } \\
\text { reported }\end{array}$ & Not reported & $\begin{array}{l}\text { Not } \\
\text { reported }\end{array}$ & Not reported & Not reported \\
\hline $\begin{array}{l}\text { Blood glucose } \\
\text { sampling }\end{array}$ & $\begin{array}{l}\text { Arterial/ } \\
\text { capillary }\end{array}$ & Arterial/capillary & $\begin{array}{c}\text { Arterial } \\
\text { preferred/ } \\
\text { capillary } \\
\text { discouraged }\end{array}$ & Not reported & $\begin{array}{l}\text { Not } \\
\text { reported }\end{array}$ & Not reported & Arterial/capillary \\
\hline Frequency of testing & $\begin{array}{c}\text { Every hour } \\
\text { until stable, } \\
\text { then every } \\
2-4 \mathrm{~h}\end{array}$ & Every $1-4 \mathrm{~h}$ & Every hour & Every $6 \mathrm{~h}$ & $\begin{array}{l}\text { Not } \\
\text { reported }\end{array}$ & Every $4 \mathrm{~h}$ & $\begin{array}{l}\text { Every } 1-4 \mathrm{~h} \text { on } \\
\text { insulin; } \\
\text { every } 4-6 \mathrm{~h} \text { if no } \\
\text { insulin }\end{array}$ \\
\hline Type of insulin & $\begin{array}{l}\text { Intravenous } \\
\text { insulin }\end{array}$ & $\begin{array}{l}\text { Intravenous } \\
\text { insulin, used } \\
\text { same protocol } \\
\text { as Leuven } \\
\text { study }\end{array}$ & $\begin{array}{l}\text { Intravenous } \\
\text { insulin }\end{array}$ & $\begin{array}{c}\text { Intravenous } \\
\text { insulin, } \\
\text { intense; } \\
\text { subcutaneous, } \\
\text { control }\end{array}$ & $\begin{array}{l}\text { Not } \\
\text { reported }\end{array}$ & $\begin{array}{l}\text { Intravenous } \\
\text { insulin }\end{array}$ & $\begin{array}{l}\text { Intravenous } \\
\text { insulin }\end{array}$ \\
\hline $\begin{array}{l}\text { Duration of study } \\
\text { intervention }\end{array}$ & $\begin{array}{l}\text { Duration } \\
\text { of ICU stay }\end{array}$ & $\begin{array}{c}\text { Duration of ICU } \\
\text { stay }\end{array}$ & $\begin{array}{l}\text { Duration of } \\
\text { ICU stay } \\
\text { or stopped } \\
\text { when eating }\end{array}$ & Not reported & $\begin{array}{l}\text { Not } \\
\text { reported }\end{array}$ & $\begin{array}{c}\text { Duration of ICU } \\
\text { stay }\end{array}$ & $\begin{array}{c}\text { Duration of ICU } \\
\text { stay }\end{array}$ \\
\hline Feeding & $\begin{array}{l}\text { Enteral or } \\
\text { parenteral }\end{array}$ & $\begin{array}{c}40-50 \% \\
\text { given enteral }\end{array}$ & $\begin{array}{l}\text { Enteral/ } \\
\text { parenteral/ } \\
\text { glucose }\end{array}$ & Not reported & $\begin{array}{l}\text { Not } \\
\text { reported }\end{array}$ & $\begin{array}{c}\text { Enteral/ } \\
\text { parenteral } \\
\text { as soon as } \\
\text { possible } 25 \\
\mathrm{kcal} / \mathrm{kg} \text { ideal } \\
\text { body weight }\end{array}$ & $\begin{array}{l}95 \% \text { enteral, } \\
5 \% \text { enteral and } \\
\text { parenteral. } \\
\text { If neither, } 10 \% \\
\text { dextrose } 5 \mathrm{~g} / \mathrm{h}\end{array}$ \\
\hline $\begin{array}{l}\text { Target blood glucose, } \\
\text { intensive }(\mathrm{mg} / \mathrm{dl})\end{array}$ & $80-110$ & $80-110$ & $81-108$ & $80-120$ & $80-110$ & $80-110$ & $80-110$ \\
\hline $\begin{array}{l}\text { Target blood glucose, } \\
\text { control (mg/dl) }\end{array}$ & $180-200$ & $180-200$ & $144-180$ & $<180$ & $140-180$ & $180-200$ & $180-200$ \\
\hline $\begin{array}{l}\text { Mean blood glucose, } \\
\text { intensive (mg/dl) }\end{array}$ & 115 & 112 & $\begin{array}{c}\text { Time } \\
\text { weighted } \\
115\end{array}$ & 134 & 119 & 110 & 120 \\
\hline
\end{tabular}


Table 2. Continued

\begin{tabular}{|c|c|c|c|c|c|c|c|}
\hline & Arabi et al. ${ }^{29}$ & $\begin{array}{l}\text { Brunkhorst } \\
\text { et al. }{ }^{31 a}\end{array}$ & NICE-SUGAR ${ }^{34}$ & $\begin{array}{l}\text { Azevedo } \\
\text { et al. }{ }^{30}\end{array}$ & Devos et al. ${ }^{33}$ & lapichino et al. ${ }^{35}$ & $\begin{array}{c}\text { De La Rosa Gdel } \\
\text { et al. }{ }^{32}\end{array}$ \\
\hline $\begin{array}{l}\text { Mean blood } \\
\text { glucose, } \\
\text { control (mg/dl) }\end{array}$ & $\begin{array}{c}171 \\
p<.0001\end{array}$ & 151 & $\begin{array}{c}\text { Time weighted } \\
144\end{array}$ & $\begin{array}{c}144, \\
p=.003\end{array}$ & $\begin{array}{c}147 \\
p<.0001\end{array}$ & $\begin{array}{c}163 \\
p<.0001\end{array}$ & $\begin{array}{c}149 \\
p<.001\end{array}$ \\
\hline APACHE-II & 23 & $\sim 20$ & 21 & Not reported & 17 & Not reported & 16 \\
\hline $\begin{array}{l}\text { Primary } \\
\text { endpoint }\end{array}$ & ICU mortality ${ }^{b}$ & $\begin{array}{l}\text { 28-day mortality } \\
\text { Sequential } \\
\text { Organ Failure } \\
\text { Assessment } \\
\text { score }\end{array}$ & $\begin{array}{l}\text { 28-day and } 90 \\
\text { day mortality }\end{array}$ & Not reported & ICU mortality & $\begin{array}{c}\text { Asymmetric, } \\
\text { dimetthylarginine }^{\text {values }}{ }^{c}\end{array}$ & 28-day mortality \\
\hline $\begin{array}{l}\text { Mortality, } \\
\text { intensive }\end{array}$ & $\begin{array}{l}\text { ICU }(13.5 \%) ; \\
\text { hospital, } \\
72(27.1 \%) ; \\
\text { medical } \\
\text { patients only, } \\
33 / 223(15 \%)\end{array}$ & $\begin{array}{c}28 \text { days, } 61 / 247 \\
(25 \%) ; \\
90 \text { days, } \\
98 / 247(40 \%)\end{array}$ & $\begin{array}{c}28 \text { days, } \\
670 / 3010 \\
(22 \%) ; \\
90 \text { days, } \\
829 / 3010 \\
(27.5 \%)\end{array}$ & $\begin{array}{l}\text { ICU }(22.6 \%) \\
>5 \text {-day stay } \\
\quad(26 \%)\end{array}$ & ICU (16.7\%) & $\begin{array}{c}\text { ICU, } 8(22 \%) ; \\
90 \text { days, } 13 \\
(36 \%)\end{array}$ & $\begin{array}{c}\text { ICU, } 84 / 250 \\
(33 \%) \text { relative } \\
\text { risk } 1.06 ; \\
28 \text { days, 93/254 } \\
(37 \%) \text { relative } \\
\text { risk } 1.1 ; \\
\text { hospital } \\
102 / 254(40 \%) \\
\text { relative risk } 1.05\end{array}$ \\
\hline $\begin{array}{l}\text { Mortality, } \\
\text { control }\end{array}$ & $\begin{array}{c}\text { ICU }(17 \%) \\
p=.3 ; \\
\text { hospital, } 83 \\
\text { (32\%), } p=.19 ; \\
\text { medical } \\
\text { patients only, } \\
39 / 212(18 \%) \\
p=.50\end{array}$ & $\begin{array}{c}28 \text { days, } 75 / 289 \\
(26 \%), p=.74 \\
90 \text { days, } \\
102 / 288(35 \%) \\
p=.31\end{array}$ & $\begin{array}{c}28 \text { days, } \\
627 / 3012 \\
(21 \%), \\
p=.17 ; \\
90 \text { days, } \\
751 / 3012 \\
(25 \%), \\
p=.002\end{array}$ & $\begin{array}{c}\text { ICU, 25\%, } \\
p=.6 ; \\
>5-\text { day stay } \\
(30 \%) \text { NS }^{d}\end{array}$ & $\begin{array}{c}\text { ICU, } \\
15 \% \mathrm{NS}^{d}\end{array}$ & $\begin{array}{c}\text { ICU, } 6(17 \%), \\
p=.55 \\
90 \text { days, } \\
11(31 \%) \\
p=.62\end{array}$ & $\begin{array}{c}\text { ICU, } 78 / 250 \\
\text { (31\%); } \\
28 \text { days, } \\
\text { 81/250 (32\%); } \\
\text { hospital, } \\
96 / 250(38 \%)\end{array}$ \\
\hline $\begin{array}{l}\text { Mean LOS, } \\
\text { intensive (days) }\end{array}$ & ICU, 10 & ICU, 16 & $\begin{array}{c}\text { ICU, 6; } \\
\text { hospital, } 17\end{array}$ & Not reported & Not reported & 16 & ICU, 6 \\
\hline $\begin{array}{l}\text { Mean LOS, } \\
\text { control (days) }\end{array}$ & $\begin{array}{c}\text { ICU, } 11 \\
p=.18\end{array}$ & $\begin{array}{l}\text { ICU, 14, } \\
p=.06\end{array}$ & $\begin{array}{c}\text { ICU, 6; } \\
\text { hospital, } 17\end{array}$ & Not reported & Not reported & $\begin{array}{c}13 \\
p=.74\end{array}$ & ICU, 6 \\
\hline $\begin{array}{l}\text { Hypoglycemic } \\
\text { events }<40 \\
\mathrm{mg} / \mathrm{dl}(\%), \\
\text { intensive }\end{array}$ & $\begin{array}{c}76 \\
(29 \%)\end{array}$ & $\begin{array}{l}42 / 247 \\
(17 \%)\end{array}$ & $\begin{array}{c}206 / 3016 \\
(7 \%)\end{array}$ & $\begin{array}{c}27 \\
(16 \%)\end{array}$ & $(10 \%)$ & $8(22 \%)$ & $21(8 \%)$ \\
\hline $\begin{array}{l}\text { Hypoglycemic } \\
\text { events < } 40 \\
\text { mg/dl, control }\end{array}$ & $\begin{array}{c}8(3 \%) \\
p<.0001\end{array}$ & $\begin{array}{c}12 / 290(4 \%) \\
p<.001\end{array}$ & $\begin{array}{c}15 / 3014(0.5 \%) \\
p<.001\end{array}$ & $\begin{array}{c}6(4 \%) \\
p<.001\end{array}$ & $\begin{array}{c}3 \% \\
p<.0001\end{array}$ & $\begin{array}{c}3(8 \%), \\
p=.189\end{array}$ & $\begin{array}{c}2(2 \%) \\
p<.001\end{array}$ \\
\hline $\begin{array}{l}\text { Hypoglycemic } \\
\text { events } 40-60 \\
\mathrm{mg} / \mathrm{dl}(\%), \\
\text { intense }\end{array}$ & Not studied & Not studied & Not studied & $\begin{array}{l}\text { Hypoglycemia } \\
\text { not defined }\end{array}$ & Not studied & Not studied & $66 \%$ \\
\hline $\begin{array}{l}\text { Hypoglycemic } \\
\text { events } 40-60 \\
\mathrm{mg} / \mathrm{dl}(\%), \\
\text { control }\end{array}$ & Not studied & Not studied & Not studied & $\begin{array}{l}\text { Hypoglycemia } \\
\text { not defined }\end{array}$ & Not studied & Not studied & $10 \%$ \\
\hline \multicolumn{8}{|c|}{$\begin{array}{l}\text { a Stopped early (after } 488 \text { patients) because of increased incidence of hypoglycemia. } \\
b \text { Those with APACHE } \leq 22 \text { and body mass index } \leq 26.2 \text { showed decreased in mortality with IIT. Those with Glasgow Coma Scale } \leq 9 \text { noted } \\
\text { to have increased mortality with IIT. } \\
\text { c Product released during physiologic protein turnover, high levels associated with endothelial dysfunction, oxidative injury, and } \\
\text { inflammation. Strong predictor of mortality in critically ill patients. } \\
\text { d }\end{array}$} \\
\hline
\end{tabular}




\section{Impact of Diabetes on Outcomes}

The NICE-SUGAR trial investigators performed a subgroup analysis $^{34}$ of patients with and without diabetes. In this study, a higher percentage of individuals with diabetes died at 90 days compared to patients without diabetes in both intensively treated and control groups (31.7\% versus $26.4 \%$ and $27.6 \%$ versus $24.2 \%$, respectively). The OR for death for diabetes patients was 1.21 (95\% CI, 0.95 to 1.55$)$ and for nondiabetes patients $1.12(95 \% \mathrm{CI}$, 0.99 to 1.28), with a nonsignificant $p$ value for heterogeneity of 0.6 , meaning that there was no significant difference in treatment effect between those with and without diabetes. Some additional studies that have attempted get to the bottom of this issue suggest that the relationship between hyperglycemia and mortality is different in critically ill patients with diabetes versus without diabetes. In a retrospective observational study, ${ }^{36}$ patients without known diabetes had higher ICU and hospital mortality compared to those with diabetes for blood glucose ranges $<80,144-180,180-200$, and $>200 \mathrm{mg} / \mathrm{dl}$. Another case-control study ${ }^{37}$ showed that, for any given insulin dose, mortality was higher in patients without diabetes than individuals with diabetes. Mortality increased for patients without diabetes at $144 \mathrm{mg} / \mathrm{dl}$ and for patients with diabetes at $200 \mathrm{mg} / \mathrm{dl}$. Individuals with diabetes were sicker with higher glucose levels, but their mortality was the same as controls who didn't require insulin. Persons without diabetes who had hyperglycemia and required insulin had mortality rates twice as high as those with diabetes and controls. Both of these studies evaluated mixed ICU populations, but similar results have been shown in isolated critically ill populations such as those suffering from myocardial infarction and acute stroke. ${ }^{36,37}$ A meta-analysis showed that the pooled relative risk of in-hospital mortality after myocardial infarction in 1856 patients without diabetes who had stress hyperglycemia on admission was 3.9, and 688 patients with diabetes who had stress hyperglycemia on admission had a pooled unadjusted relative risk of 1.7..$^{38}$ A study by Kosiborod and coworkers ${ }^{39}$ evaluated patients greater than 65 years old and noted that those with diabetes had a higher mortality at glucose levels $<110 \mathrm{mg} / \mathrm{dl}$ for 30-day mortality and $<170 \mathrm{mg} / \mathrm{dl}$ for 1 year mortality. However, patients without diabetes had higher mortality above these levels. Capes and associates also performed a meta-analysis in acute stroke patients, which showed an unadjusted relative risk of in-hospital or 30-day morality to be 3.07 (95\% CI, 2.5 to 3.79) for patients without diabetes and $1.30(95 \% \mathrm{CI}, 0.49$ to 3.43$)$ for those with diabetes. ${ }^{40}$ Results of longer-term follow-up (3 months to 1 year) were mixed, with only 5 out of 10 studies associating stress hyperglycemia with increased mortality, but did seem to associate poor functional recovery up to 6 months after acute stroke with persons without diabetes who had stress hyperglycemia. Two additional studies assessing outcomes in acute stroke showed that admission hyperglycemia ${ }^{41}$ and prolonged stress hyperglycemia ${ }^{42}$ predict poorer prognosis and increase mortality, especially in patients without diabetes.

\section{Assessment of Different Insulin Infusion Protocols}

Since IIT has taken center stage, many different insulin protocols have been developed that attempt to attain normoglycemia and avoid hypoglycemia. Prior to this new era of glycemic control, insulin infusion was initiated in critically ill patients and titrated by nurses without much direction as to how to accomplish whatever goal was needed, or perhaps there was no true goal. Upon development and implementations of insulin infusion protocols, it has been shown by many that they have been able to safely and effectively achieve goal blood glucose. ${ }^{43-48}$ An attempt to compare different protocols was difficult because of the many differences between protocols. ${ }^{49}$ Protocols or nomograms guiding insulin dosing should work to achieve glycemic control quickly with minimal hypoglycemia, have low operator error rate, and minimize nursing time required. The best protocols adjust for direction and rate of glucose decline, permit "off protocol" adjustments, and take individuality of patients into account. ${ }^{49}$

\section{Cost-Effectiveness Studies}

There are few studies looking at cost-effectiveness of insulin therapy in the ICU. Van den Berghe and coworkers performed a cost analysis for their surgical ICU study ${ }^{50}$ and demonstrated a cost savings of 2638 Euro per patient in the IIT group. A mixed medical-surgical ICU nonrandomized study estimated a reduction in length of stay (LOS) by 0.3 days and a cost savings of $\$ 1580$ per patient for the entire hospitalization. ${ }^{51}$ An additional singlecenter study compared cost for five subspecialty ICUs using protocolized IIT to four other subspecialty ICUs managing hyperglycemia according to the discretion of the individual physicians. ${ }^{52}$ The intervention was associated with a trend toward shorter LOS, lower costs (reduction of \$5231 in total ICU costs), and lower mortality. Intensive insulin therapy and monitoring certainly is more expensive than conventional therapy and monitoring. The cost analyses that have occurred took place in single centers and showed reduction in mortality, LOS, 
and/or morbidity with IIT, which then resulted in a substantially lower overall cost than conventional treatment. The NICE-SUGAR study did not confirm benefits of intensive control on mortality, morbidity, or $\mathrm{LOS}^{34}$ so it would be important to reassess costeffectiveness of controlling hyperglycemia in the critically ill population.

\section{Clinical Implications}

The surgical ICU study of Van den Berghe and colleagues caused the glycemic pendulum to swing toward intensive control since its publication in 2001. ${ }^{1}$ Tight glycemic control has become a quality measure, and perhaps this study, despite its critics, brought the intensive care community to the realization that hyperglycemia (stress or otherwise) should not be ignored. But the release of the NICE-SUGAR study ${ }^{34}$ is creating a lot of discussion, and perhaps the pendulum will be swinging away from normoglycemia in the ICU. The professional societies have quickly changed their guidelines for glycemic goals in the ICU to be $140-180 \mathrm{mg} / \mathrm{dl}$ and on the general medical wards between $100-180 \mathrm{mg} / \mathrm{dl} .{ }^{53}$ From a practical perspective, key take home points are as follows: (1) Do not neglect glycemic control in critically ill patients, as studies have tested not tight versus no/poor control, but tight versus good control. The former question needs to be answered, but until then, summative evidence would have us believe that reduction of severe hyperglycemia would reduce morbidity, especially from infectious complications. (2) There is no additional benefit in outcomes obtained by achieving normoglycemia compared to reasonable glycemic control. A moderate approach to managing critical illness hyperglycemia seems most prudent at this juncture. The ADA has appropriately altered guidelines to aim for glucose of $140-180 \mathrm{mg} / \mathrm{dl}$ for critically ill patients, and ICUs should make efforts to adjust to these goals. (3) There is harm from overt and quite possibly unrecognized hypoglycemia with IIT. Thus strict goals cannot be achieved safely given the limitations of current technology in monitoring glucose levels. It remains to be seen if there is benefit of tight glucose control once hypoglycemia is minimized with technological advances such as continuous glucose monitoring systems.

\section{Future Directions}

Certainly, the underlying pathophysiologic mechanisms behind stress hyperglycemia need to be characterized further to attempt to prevent its adverse consequences. While glycemic goals have been set in a moderate range, it may be that certain subsets of patients would benefit from different glycemic goals. Further studies will need to be completed to determine whether there is a need to individualize glycemia depending on the patient and their type of critical illness. Future research should also focus on hospital hyperglycemia outside of the ICUs, on the general medical-surgical floors, where patients in this day and age can still be found with glucose levels greater than $200 \mathrm{mg} / \mathrm{dl}$ being treated with sliding scale insulin.

\section{References:}

1. Van den Berghe G, Wouters P, Weekers F, Verwaest C, Bruyninckx F, Schetz M, Vlasselaers D, Ferdinande P, Lauwers P, Bouillon R. Intensive insulin therapy in the critically ill patients. New Engl J Med. 2001;345(19):1359-67.

2. Cely CM, Arora P, Quartin AA, Kett DH, Schein RM. Relationship of baseline glucose homeostasis to hyperglycemia during medical critical illness. Chest. 2004;126(3):879-87.

3. Gandhi GY, Nuttall GA, Abel MD, Mullany CJ, Schaff HV, O'Brien PC, Johnson MG, Williams AR, Cutshall SM, Mundy LM, Rizza RA, McMahon MM. Intensive intraoperative insulin therapy versus conventional glucose management during cardiac surgery: a randomized trial. Ann Intern Med. 2007;146(4):233-43.

4. Dungan KM, Braithwaite SS, Preiser JC. Stress hyperglycaemia Lancet. 2009;373(9677):1798-807.

5. Blumberg D, Hochwald S, Burt M, Donner D, Brennan MF Tumor necrosis factor alpha stimulates gluconeogenesis from alanine in vivo. J Surg Oncol. 1995;59(4):220-4.

6. Fan J, Li $\mathrm{YH}$, Wojnar MM, Lang $\mathrm{CH}$. Endotoxin-induced alterations in insulin-stimulated phosphorylation of insulin receptor, IRS-1, and MAP kinase in skeletal muscle. Shock. 1996;6(3):164-70.

7. Gamelli RL, Liu H, He LK, Hofmann CA. Augmentations of glucose uptake and glucose transporter-1 in macrophages following thermal injury and sepsis in mice. J Leukoc Biol. 1996;59(5):639-47.

8. Lang $\mathrm{CH}$, Dobrescu C. Gram-negative infection increases noninsulinmediated glucose disposal. Endocrinology. 1991;128(2):645-53.

9. Shamoon H, Hendler R, Sherwin RS. Altered responsiveness to cortisol, epinephrine, and glucagon in insulin-infused juvenileonset diabetics. A mechanism for diabetic instability. Diabetes. 1980;29(4):284-91.

10. McMahon MM, Rizza RA. Nutrition support in hospitalized patients with diabetes mellitus. Mayo Clin Proc. 1996;71(6):587-94.

11. Montori VM, Bistrian BR, McMahon MM. Hyperglycemia in acutely ill patients. JAMA. 2002;288(17):2167-9.

12. Stuart CA, Shangraw RE, Prince MJ, Peters EJ, Wolfe RR. Bed-rest-induced insulin resistance occurs primarily in muscle. Metabolism. 1988;37(8):802-6. 
13. Clement S, Braithwaite SS, Magee MF, Ahmann A, Smith EP, Schafer RG, Hirsch IB, American Diabetes Association Diabetes in Hospitals Writing Committee. Management of diabetes and hyperglycemia in hospitals. Diabetes Care. 2004;27(2):553-91.

14. Kwoun MO, Ling PR, Lydon E, Imrich A, Qu Z, Palombo J, Bistrian BR. Immunologic effects of acute hyperglycemia in nondiabetic rats. JPEN J Parenter Enteral Nutr. 1997;21(2):91-5.

15. Gustafson KS, Vercellotti GM, Bendel CM, Hostetter MK. Molecular mimicry in Candida albicans. Role of an integrin analogue in adhesion of the yeast to human endothelium. J Clin Invest. 1991;87(6):1896-902.

16. Hostetter MK. Handicaps to host defense. Effects of hyperglycemia on C3 and Candida albicans. Diabetes. 1990;39(3):271-5.

17. Hostetter MK. The third component of complement: new functions for an old friend. J Lab Clin Med. 1993;122(5):491-6.

18. Cohen G, Riahi Y, Alpert E, Gruzman A, Sasson S. The roles of hyperglycaemia and oxidative stress in the rise and collapse of the natural protective mechanism against vascular endothelial cell dysfunction in diabetes. Arch Physiol Biochem. 2007;113(4-5):259-67.

19. Brownlee M. The pathobiology of diabetic complications: a unifying mechanism. Diabetes. 2005;54(6):1615-25.

20. Koya D, Jirousek MR, Lin YW, Ishii H, Kuboki K, King GL. Characterization of protein kinase $\mathrm{C}$ beta isoform activation on the gene expression of transforming growth factor-beta, extracellular matrix components, and prostanoids in the glomeruli of diabetic rats. J Clin Invest. 1997;100(1):115-26.

21. Vanhorebeek I, Van den Berghe G. Diabetes of injury: novel insights. Endocrinol Metab Clin North Am. 2006;35(4):859-72.

22. Bruno A, Kent TA, Coull BM, Shankar RR, Saha C, Becker KJ Kissela BM, Williams LS. Treatment of hyperglycemia in ischemic stroke (THIS): a randomized pilot trial. Stroke. 2008;39(2):384-9.

23. Walters MR, Weir CJ, Lees KR. A randomised, controlled pilot study to investigate the potential benefit of intervention with insulin in hyperglycaemic acute ischaemic stroke patients. Cerebrovasc Dis. 2006;22(2-3):116-22.

24. Oksanen T, Skrifvars MB, Varpula T, Kuitunen A, Pettilä V, Nurmi J, Castrén M. Strict versus moderate glucose control after resuscitation from ventricular fibrillation. Intensive Care Med. 2007;33(12):2093-100.

25. Farah R, Samokhvalov A, Zviebel F, Makhoul N. Insulin therapy of hyperglycemia in intensive care. Isr Med Assoc J. 2007;9(3):140-2.

26. Van den Berghe G, Wilmer A, Hermans G, Meersseman W, Wouters PJ, Milants I, Van Wijngaerden E, Bobbaers H, Bouillon R. Intensive insulin therapy in the medical ICU. New Engl J Med. 2006;354(5):449-61.

27. Bland DK, Fankhanel Y, Langford E, Lee M, Lee SW, Maloney C, Rogers M, Zimmerman G. Intensive versus modified conventional control of blood glucose level in medical intensive care patients: a pilot study. Am J Crit Care. 2005;14(5):370-6.

28. Griesdale DE, de Souza RJ, van Dam RM, Heyland DK, Cook DJ, Malhotra A, Dhaliwal R, Henderson WR, Chittock DR, Finfer S, Talmor D. Intensive insulin therapy and mortality among critically ill patients: a meta-analysis including NICE-SUGAR study data. CMAJ. 2009;180(8):821-7.

29. Arabi YM, Dabbagh OC, Tamim HM, Al-Shimemeri AA, Memish ZA, Haddad SH, Syed SJ, Giridhar HR, Rishu AH, Al-Daker MO, Kahoul SH, Britts RJ, Sakkijha MH. Intensive versus conventional insulin therapy: a randomized controlled trial in medical and surgical critically ill patients. Crit Care Med. 2008;36(12):3190-7.

30. Azevedo JR, de Araujo LO, Azevedo RP, Silva WS, Cruz FL. Intensive insulin therapy versus glycemic control in critically ill patients: a prospective controlled trial [abstract]. Crit Care Med. 2007;11(Suppl 3):P82.
31. Brunkhorst FM, Engel C, Bloos F, Meier-Hellmann A, Ragaller M, Weiler $\mathrm{N}$, Moerer $\mathrm{O}$, Gruendling $\mathrm{M}$, Oppert $\mathrm{M}$, Grond $\mathrm{S}$, Olthoff D, Jaschinski U, John S, Rossaint R, Welte T, Schaefer M, Kern P, Kuhnt E, Kiehntopf M, Hartog C, Natanson C, Loeffler M, Reinhart K, German Competence Network Sepsis (SepNet). Intensive insulin therapy and pentastarch resuscitation in severe sepsis. N Engl J Med. 2008;358(2):125-39.

32. De La Rosa Gdel C, Donado JH, Restrepo AH, Quintero AM, González LG, Saldarriaga NE, Bedoya M, Toro JM, Velásquez JB, Valencia JC, Arango CM, Aleman PH, Vasquez EM, Chavarriaga JC, Yepes A, Pulido W, Cadavid CA, Grupo de Investigacion en Cuidado intensivo: GICI-HPTU. Strict glycaemic control in patients hospitalised in a mixed medical and surgical intensive care unit: a randomised clinical trial. Crit Care. 2008;12(5):R120.

33. Devos P, Preiser J, Melot C. Impact of tight glucose control by intensive insulin therapy on ICU mortality and the rate of hypoglycaemia: final results of the glucontrol study. Intensive Care Med. 2007;33(Suppl 2):S189.

34. NICE-SUGAR Study Investigators, Finfer S, Chittock DR, Su SY, Blair D, Foster D, Dhingra V, Bellomo R, Cook D, Dodek P, Henderson WR, Hébert PC, Heritier S, Heyland DK, McArthur C, McDonald E, Mitchell I, Myburgh JA, Norton R, Potter J, Robinson BG, Ronco JJ. Intensive versus conventional glucose control in critically ill patients. N Engl J Med. 2009;360(13):1283-97.

35. Iapichino $G$, Albicini $M$, Umbrello $M$, Sacconi $F$, Fermo I, Pavlovich R, Paroni R, Bellani G, Mistraletti G, Cugno M, Pesenti A, Gattinoni L. Tight glycemic control does not affect asymmetric-dimethylarginine in septic patients. Intensive Care Med. 2008;34(10):1843-50.

36. Egi M, Bellomo R, Stachowski E, French CJ, Hart GK, Hegarty C, Bailey M. Blood glucose concentration and outcome of critical illness: the impact of diabetes. Crit Care Med. 2008;36(8):2249-55.

37. Rady MY, Johnson DJ, Patel BM, Larson JS, Helmers RA. Influence of individual characteristics on outcome of glycemic control in intensive care unit patients with or without diabetes mellitus. Mayo Clin Proc. 2005;80(12):1558-67.

38. Capes SE, Hunt D, Malmberg K, Gerstein HC. Stress hyperglycaemia and increased risk of death after myocardial infarction in patients with and without diabetes: a systematic overview. Lancet. 2000;355(9206):773-8.

39. Kosiborod M, Rathore SS, Inzucchi SE, Masoudi FA, Wang Y, Havranek EP, Krumholz HM. Admission glucose and mortality in elderly patients hospitalized with acute myocardial infarction: implications for patients with and without recognized diabetes. Circulation. 2005;111(23):3078-86.

40. Capes SE, Hunt D, Malmberg K, Pathak P, Gerstein HC. Stress hyperglycemia and prognosis of stroke in nondiabetic and diabetic patients: a systematic overview. Stroke. 2001;32(10):2426-32.

41. Stöllberger C, Exner I, Finsterer J, Slany J, Steger C. Stroke in diabetic and non-diabetic patients: course and prognostic value of admission serum glucose. Ann Med. 2005;37(5):357-64.

42. Kes VB, Solter VV, Supanc V, Demarin V. Impact of hyperglycemia on ischemic stroke mortality in diabetic and non-diabetic patients. Ann Saudi Med. 2007;27(5):352-5.

43. Brown G, Dodek P. Intravenous insulin nomogram improves blood glucose control in the critically ill. Crit Care Med. 2001;29(9):1714-9.

44. Davidson PC, Steed RD, Bode BW. Glucommander: a computerdirected intravenous insulin system shown to be safe, simple, and effective in 120,618 h of operation. Diabetes Care. 2005;28(10):2418-23.

45. Goldberg PA, Siegel MD, Sherwin RS, Halickman JI, Lee M, Bailey VA, Lee SL, Dziura JD, Inzucchi SE. Implementation of a safe and effective insulin infusion protocol in a medical intensive care unit. Diabetes Care. 2004;27(2):461-7. 
46. Juneja R, Roudebush C, Kumar N, Macy A, Golas A, Wall D, Wolverton C, Nelson D, Carroll J, Flanders SJ. Utilization of a computerized intravenous insulin infusion program to control blood glucose in the intensive care unit. Diabetes Technol Ther. 2007;9(3):232-40.

47. Kanji S, Singh A, Tierney M, Meggison H, McIntyre L, Hebert PC. Standardization of intravenous insulin therapy improves the efficiency and safety of blood glucose control in critically ill adults. Intensive Care Med. 2004;30(5):804-10.

48. Thomas AN, Marchant AE, Ogden MC, Collin S. Implementation of a tight glycaemic control protocol using a web-based insulin dose calculator. Anaesthesia. 2005;60(11):1093-100.

49. Wilson M, Weinreb J, Hoo GW. Intensive insulin therapy in critical care: a review of 12 protocols. Diabetes Care. 2007;30(4):1005-11.

50. Van den Berghe G, Wouters PJ, Kesteloot K, Hilleman DE. Analysis of healthcare resource utilization with intensive insulin therapy in critically ill patients. Crit Care Med. 2006;34(3):612-6.

51. Krinsley JS, Jones RL. Cost analysis of intensive glycemic control in critically ill adult patients. Chest. 2006;129(3):644-50.

52. Sadhu AR, Ang AC, Ingram-Drake LA, Martinez DS, Hsueh WA, Ettner SL. Economic benefits of intensive insulin therapy in critically ill patients: the targeted insulin therapy to improve hospital outcomes (TRIUMPH) project. Diabetes Care. 2008;31(8):1556-61.

53. Moghissi ES, Korytkowski MT, DiNardo M, Einhorn D, Hellman R, Hirsch IB, Inzucchi SE, Ismail-Beigi F, Kirkman MS, Umpierrez GE, American Association of Clinical Endocrinologists, American Diabetes Association. American Association of Clinical Endocrinologists and American Diabetes Association consensus statement on inpatient glycemic control. Diabetes Care. 2009;32(6):1119-31. 